\title{
NEW MEMBERSHIP CRITERIA FOR 437 FLARE STARS IN THE PLEIADES
}

\author{
G. SZÉCSÉNYI-NAGY \\ Eötvös University, Department of Astronomy, Budapest, Hungary \\ szena@ludens.elte.hu \\ AND \\ E. SCHILBACH, S. HIRTE AND R.-D. SCHOLZ \\ Astrophysikalisches Institut Potsdam, Potsdam, Germany \\ eschilbach@aip.de, shirte@aip.de and rdscholz@aip.de
}

\section{New Proper Motion Determinations and Photometry}

Most of the flare stars (FSs) discovered in that field are very faint and are missing from all previously published lists of Pleiades cluster members. Reliable membership probabilities (MPs) were only determined for one third of these objects while the rest were supposed to be more or less probable members with a few exceptions. A recent proper motion survey of that field (Schilbach et al. 1995) based on plates taken with the Tautenburg Schmidt telescope and extended to an area of 16.5 square degrees provided new cluster MPs and also photometric data even for a number of the lowest luminosity stars (in fact the faintest object measured has $V=18.26$ ). Of the 520 known FSs in the field $437(85 \%)$ were successfully identified, their J2000.0 coordinates, high precision proper motion components and apparent photographic $R$ magnitudes (and for the majority of them their $V$ and $B$ magnitudes too) determined. Based on these data new cluster MPs have been deduced which show that a considerable number (40\%) of the so called Pleiades FSs are not members of that cluster at all. This conclusion follows the tendency already found: when we involve more and more faint FSs into the investigations the percentage of non-members monotonically increases (cf. Haro et al. 1982 and references therein).

\section{The Most Important Results}

1. The number of FSs with well determined MPs has been increased by 137.5 percent in the so called Pleiades field (to $85 \%$ of the catalogued objects). 2. The statistics is even more complete in the Largest Common Field (LCF, for more details see Szécsényi-Nagy 1994) of the Pleiades (it. involves 407 FSs i.e. $91 \%$ of the LCF FSs). 3. The relative frequency of cluster member FSs amongst all FSs discovered in the region is between 0.45 and 0.50 . 4. Low activity FSs (with one or two very low amplitude flares observed) have even lower mean MPs (30\% to 33\%). 5. At least $40 \%$ of all FSs catalogued here are background or (less probably) foreground stars (which are obviously not more than one tenth of the non-members). 6. Amongst cluster member FSs no one which is fainter than absolute $R$ magnitude 11.2 is known. This means that 70 Myr old FSs are not active anymore below this brightness (and the corresponding mass) limit.

\section{References}

Haro, G., Chavira, E. \& Gonzalez, G. 1982, Bol. Obs. Ton. 3, No. 1 Schilbach, E., Robichon, N., Souchay, J. \& Guibert, J. 1995, A\&A 3, 6 Szécsényi-Nagy, G. 1994, IAU Symp. 161, 61

This research has been supported partly by the FHHER (grants: AMFK-418/96 and FKFP - 0201/1997). 